\title{
Host origin of plastid solute transporters in the first photosynthetic eukaryotes
}

\author{
Heather M Tyra ${ }^{* *}$, Marc Linka ${ }^{\star \dagger}$, Andreas PM Weber ${ }^{\dagger \neq}$ and \\ Debashish Bhattacharya*
}

Addresses: *Department of Biological Sciences and Roy J Carver Center for Comparative Genomics, 446 Biology Building, University of Iowa, Iowa City, IA 52242-1324, USA. 'Department of Plant Biology, S-336 Plant Biology Building, Michigan State University, East Lansing, Michigan 48824-1312, USA. *Current address: Institute for Plant Biochemistry, Heinrich-Heine-University, Gebäude 26.03.01, Universitätsstrasse 1, D40225 Düsseldorf, Germany.

$\ltimes$ These authors contributed equally to this work.

Correspondence: Andreas PM Weber. Email: andreas.weber@uni-duesseldrof.de. Debashish Bhattacharya. Email: debashibhattacharya@uiowa.edu

Published: 5 October 2007

Genome Biology 2007, 8:R212 (doi:10.1 I86/gb-2007-8-10-r2/2)

The electronic version of this article is the complete one and can be found online at http://genomebiology.com/2007/8/10/R2/2
Received: 22 June 2007

Revised: 23 August 2007

Accepted: 5 October 2007

\section{(c) 2007 Tyra et al.; licensee BioMed Central Ltd.}

This is an open access article distributed under the terms of the Creative Commons Attribution License (http://creativecommons.org/licenses/by/2.0), which permits unrestricted use, distribution, and reproduction in any medium, provided the original work is properly cited.

\begin{abstract}
Background: It is generally accepted that a single primary endosymbiosis in the Plantae (red, green (including land plants), and glaucophyte algae) common ancestor gave rise to the ancestral photosynthetic organelle (plastid). Plastid establishment necessitated many steps, including the transfer and activation of endosymbiont genes that were relocated to the nuclear genome of the 'host' followed by import of the encoded proteins into the organelle. These innovations are, however, highly complex and could not have driven the initial formation of the endosymbiosis. We postulate that the re-targeting of existing host solute transporters to the plastid fore-runner was critical for the early success of the primary endosymbiosis, allowing the host to harvest endosymbiont primary production.
\end{abstract}

Results: We tested this model of transporter evolution by conducting a comprehensive analysis of the plastid permeome in Arabidopsis thaliana. Of 137 well-annotated transporter proteins that were initially considered, 83 that are broadly distributed in Plantae were submitted to phylogenetic analysis. Consistent with our hypothesis, we find that $58 \%$ of Arabidopsis transporters, including all carbohydrate transporters, are of host origin, whereas only $12 \%$ arose from the cyanobacterial endosymbiont. Four transporter genes are derived from a Chlamydia-like source, suggesting that establishment of the primary plastid likely involved contributions from at least two prokaryotic sources.

Conclusion: Our results indicate that the existing plastid solute transport system shared by Plantae is derived primarily from host genes. Important contributions also came from the cyanobacterial endosymbiont and Chlamydia-like bacteria likely co-resident in the first algae. 


\section{Background}

Plastids in eukaryotes that contain chlorophyll are capable of carrying out photosynthesis, a process that converts light energy, carbon dioxide, and water into organic compounds. The evolutionary history of this organelle unfolded over a billion years ago when a previously non-photosynthetic protist engulfed and maintained a free-living cyanobacterium in its cytoplasm [1]. It is hard to over-state the importance of this ancient and extraordinarily rare primary endosymbiosis because plastids allowed the evolution of algae and the plants that form the base of the food chain for many ecosystems on Earth. Current data suggest that the primary endosymbiosis occurred once in the common ancestor of the red, green (including land plants), and glaucophyte algae, the Plantae [2-4], with the original plastid and the nuclear-encoded machinery for running the organelle spreading in subsequent cell captures to other branches of the eukaryotic tree [5-7]. The only other known case of a potential bona fide cyanobacterial primary endosymbiosis occurred relatively recently in the thecate amoeba Paulinella chromatophora $[8,9]$.

The gradualist view of evolution through mutation-selection suggests that it would have taken millions of years for the captured prokaryote to become fully integrated into the 'host' eukaryote, ultimately becoming the site not only for carbon fixation but also for other complex functions, such as lipid, isoprenoid, and amino acid biosynthesis [10]. These processes were associated with the migration of much of the cyanobacterial genome to the host nucleus and development of the complex protein import system that are key shared features among all canonical plastids [3,11,12]. A remarkable exception to the view that endosymbiosis was a gradual process of integration is offered by the katablepharid protist 'Hatena', which undergoes large-scale morphological changes following the engulfment of a green alga [13].

Regardless of whether the ancient primary endosymbiosis fostered an accelerated rate of morphological evolution in the Plantae ancestor or whether general cell morphology was unchanged as in the Paulinella example [14], one thing is clear - in the absence of rapid benefits to the host it is unlikely that the endosymbiosis would long have been sustained. Given the need for short-term survival, a key feature of early success for the endosymbiosis must have been the integration of the metabolism of the two cells. The key to this process would have been solute transporters that regulate the flux of metabolites (for example, ATP, phosphate, sugars and sugar phosphates, metal ions, and other important ions) across the organelle membranes. Controlled exchange in response to environmental factors such as changes in light intensity and trace metal availability [15-17] is decisive because the unregulated flux of metabolites would have had detrimental effects and, thereby, lowered the evolutionary fitness of the endosymbiosis. A complex system of solute transporters is in place today in extant plastids that provides the link between this organelle and the surrounding cytosol [18-20]. Here we focus on the evolutionary history of these plastid metabolite transporters to infer early events in plastid evolution.

We make two assumptions in this study. First, a system of metabolite transporters was a critical and early development in plastid evolution to supply the endosymbiont with essential nutrients and to enable the host to reap immediate benefit from photosynthetic primary production. It is unclear why the cyanobacterium that was destined to become the plastid escaped digestion in the host but this scenario has also played out in 'Hatena' and in Paulinella. Second, whereas the genome of the previously free-living cyanobacterium encoded all the transport systems required for the uptake of essential inorganic nutrients, it most likely did not harbor genes encoding transporters for the export of organic solutes to the host this would have served no obvious pre-existing purpose in the prokaryote. Precisely how the plastid solute transport system was established is unknown. One possible model involves a primarily cyanobacterial origin, in which the plastid continued to utilize its own original cyanobacterial solute transporters with their evolution over time into proteins that perform most or all currently known plastid permeome functions. An alternative model involves a host-driven solute transport system, likely derived from the vacuolar envelope that initially surrounded the endosymbiont after its engulfment [3]. And finally, both of the new partners could have contributed proteins equally to this machinery, resulting in a chimeric system composed of the most beneficial combination possible of prokaryotic and eukaryotic transporters. To determine which of these competing hypotheses best explains plastid transporter evolution, we undertook an initial bioinformatics analysis of 137 Arabidopsis thaliana solute transporters and then a detailed phylogenetic analysis of a subset of 83 conserved proteins that included available data from other Plantae. The Arabidopsis transporters are either predicted or have been shown to be chloroplast targeted and are ideal for tracking plastid permeome evolution. Using these data we demonstrate that over one-half of Plantae plastid targeted transporters are putatively of host origin whereas less than a quarter arose from the cyanobacterial endosymbiont. This suggests that the lasting contribution to the Plantae host-endosymbiont relationship with regard to the plastid solute transport system was made primarily by host genes. We also find evidence for the origin of four transporter genes or gene families from a Chlamydia-like source. This latter result raises the possibility that establishment of the ancient primary plastid may have involved contributions from at least two prokaryotic sources, perhaps explaining its singular nature. This hypothesis received substantial support from the recent finding of at least 21 genes of Chlamydia-like origin in the nuclear genome of the extremophilic red alga Cyanidioschyzon merolae [21]. 


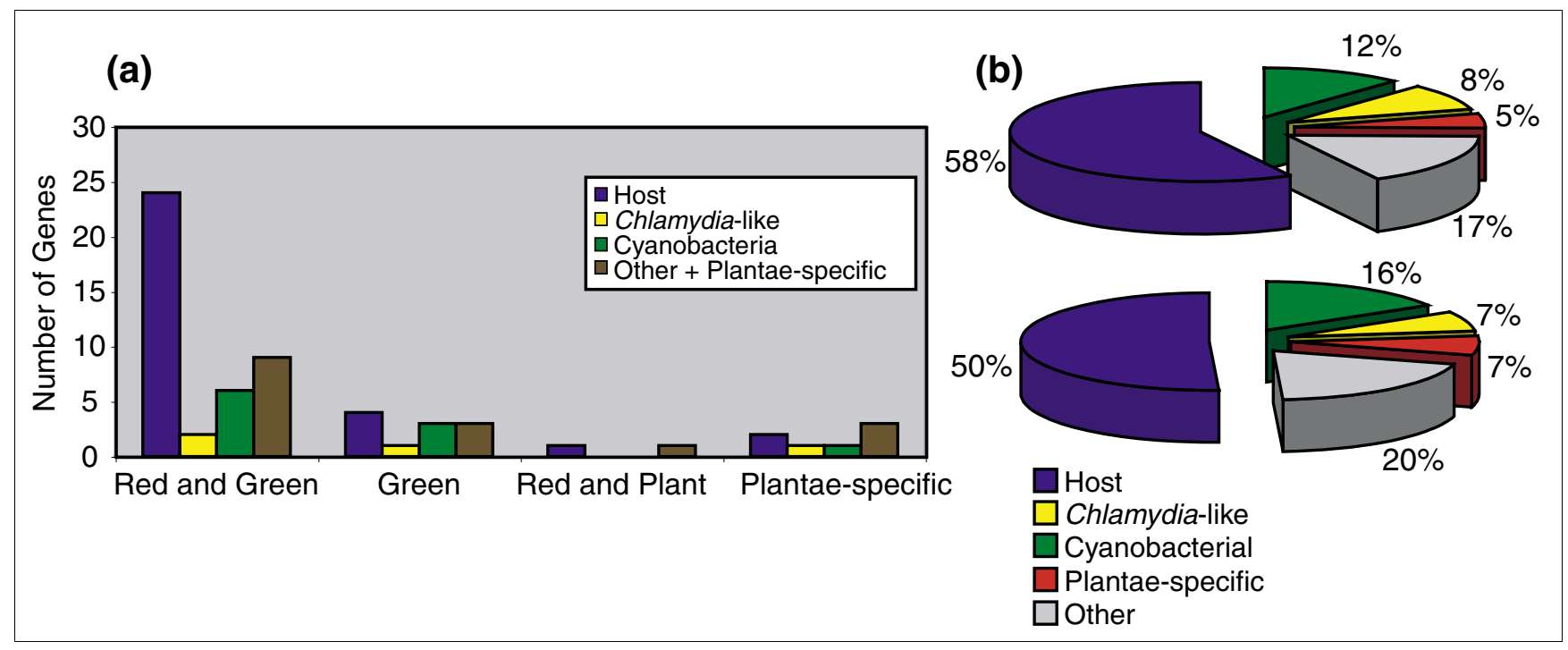

Figure I

Origin of plastid targeted solute transporters in Plantae. (a) Gene distribution among Plantae and gene origin for 63 distinct transporters considered in this study. (b) Summary pie-charts showing the origin of all the 83 transporters (top chart) and the 63 distinct genes (lower chart) considered in this study.

\section{Results and discussions}

\section{Distribution of transporters within Plantae}

Phylogenetic analysis of the best-annotated transporter data that are currently available from Arabidopsis was used to identify and putatively annotate homologs from other Plantae. Of 137 transporter proteins that were initially considered, BLAST and phylogenetic analyses and manual curation of recently available data led to the identification of 83 proteins that were of sufficient conservation and broad distribution among Plantae to be used for further analyses. Each of these 83 proteins that included gene families (that is, representing 63 distinct, ancestral genes; Table 1) was used as input in BLAST and PHYML bootstrap analyses to infer the trees. This approach identified 41 proteins that are present in both red and green algae (including land plants) and, therefore, were likely found in the Plantae ancestor (glaucophyte homologs were found for some of these genes; for example, ADP/ATP translocase, hypothetical protein At3g45890). Eleven proteins were restricted to green algae and land plants, seven were plant-specific, and two were limited to red algae and land plants. The distribution of these proteins with respect to their putative origin in Plantae is shown in Figure 1a. Given the lack of evidence for widespread horizontal gene transfer in extant Plantae, which most likely lost the capacity for phagotrophy early in its evolution $[4,22]$, we postulate that the patchy distribution for many plastid targeted transporters primarily reflects differential gene loss over the greater than one billion years that has passed since the primary endosymbiosis [1]. Under this interpretation, the large set of shared transporters among Plantae lineages provides resounding support for the monophyly of this supergroup [23].

\section{Most proteins of the plastid envelope permeome are host-derived}

Analysis of the phylogenetic data supports the notion that the host drove the integration of plastid and host metabolism. We find that the majority ( $58 \%$, when considering all 83 genes; Figure $1 \mathrm{~b}$ and Table 1 ) of the plastid solute transporters were most likely derived from existing host membrane proteins (see Figure $\mathrm{S} 1$ in Additional data file 1 for all trees). These 48 proteins are diverse in nature, including several ABC transporters, nucleotide and amino acid permeases, sulfate, potassium, magnesium, and iron transporters, and cation efflux proteins (see Figure 2 for S-adenosylmethionine carrier 1 (SAMT) and Arabidopsis thaliana folate transporter 1 (AtFOLT1) trees). Of particular interest is the finding that in addition to the members of the nucleotide-sugar/triose phosphate translocator gene family previously reported to be of host origin [3], all other carbohydrate transporters included in our analysis were derived from existing host proteins. This result strongly suggests that the host utilized existing eukaryotic transport proteins pre-adapted to this function to 'tap' into the photosynthates produced by the captured cyanobacterium. In addition, the Plantae host also provided transporters to facilitate the movement of valuable nutrients such as magnesium, potassium, iron, and phosphate into the captured prokaryote. The replacement of pre-existing cyanobacterial anion and cation transporters with host derived proteins again suggests that there was strong selection to rapidly establish control over and utilize the endosymbiont. This process was most likely accomplished by using transporters derived from the host vacuolar envelope [3]. 
Table I

\section{Arabidopsis solute transporters}

\section{Host}

At $\lg 05580$

At $\lg 54320$

At $\lg 59870$

At lg61800

Atlg64150

Atlg66950

At $\lg 70610$

At $\lg 79450$

At2g04620

At $2 \mathrm{~g} 13100$

At2g27810

At2g28070

At2g29650

At2g38060

At $2 \mathrm{~g} 38330$

At $2 \mathrm{~g} 40420$

At3g01550

At3g 12740

At3g 17690

At3g 17700

At3g45890

At3g52310

At4g00370

At4g13590

At4g 17340

At4g25750

At4g 32400

At $4 \mathrm{~g} 32650$

At4g 38380

At4g39460

At5g04770

At5g05630

At5g 13550

At5g 14040

At5g16150

At5g 17630

At5g19410

At5g 19600

At5g22830

At5g 26820

At5g 33320

At5g42130

At5g 45450

At5g46110

At5g52860

At5g54800

At5g59250
Cation/hydrogen exchanger

Ligand-effect modulator 3 (LEM3) family

$A B C$ transporter

Glucose-6-phosphate/phosphate translocator 2 (GPT2)

Expressed protein

$A B C$ transporter

Transporter associated with antigen processing protein I (AtTAPI)

Ligand-effect modulator 3 (LEM3) family

Cation efflux family protein

Glycerol-3-phosphate transporter

Xanthine/uracil permease

$A B C$ transporter

$\mathrm{Na}$-dependent inorganic phosphate cotransporter

$\mathrm{Na}+$-dependent inorganic phosphate cotransporter

Multi antimicrobial extrusion (MATE Efflux) protein

Amino acid transporter

Phosphoenolpyruvate/phosphate translocator 2 (PPT2)

Ligand-effect modulator 3 LEM3 family

Cyclic nucleotide-binding transporter 2

Cyclic nucleotide-binding transporter I

Expressed protein

$A B C$ transporter

Anion transporter 2 (ANTR2)

Expressed protein

Major intrinsic family protein

$A B C$ transporter

Adenine nucleotide uniporter

Arabidopsis thaliana $\mathrm{K}^{+}$rectifying channel I (ATKCI)

Multi antimicrobial extrusion (MATE Efflux) protein

S-adenosylmethionine carrier 2 (SAMT)

Amino acid permease

Amino acid permease

Sulfate transporter

Mitochondrial phosphate transporter

Hexose transporter

Glucose-6-phosphate transporter I (XPT)

$A B C$ transporter (White)

Sulfate transporter

CorA-like magnesium transporter

Ferroportin-related protein

Phosphoenolpyruvate/phosphate translocator (PPTI)

Mitochondrial substrate carrier family

Iron transporter-related

Triose phosphate translocator (TPT)

$A B C$ transporter (White)

Glucose-6-phosphate/phosphate transporter I (GPTI)

Sugar transporter 
Table I (Continued)

\begin{tabular}{|c|c|}
\hline \multicolumn{2}{|c|}{ Arabidopsis solute transporters } \\
\hline At lg04570 & Integral membrane family protein \\
\hline Atlg08640 & Expressed protein \\
\hline At $\lg 19800$ & Trigalactosyldiacylglycerol I, TGDI \\
\hline At $\lg 78620$ & Integral membrane family protein \\
\hline At2g32040 & Folate monoglutamate transporter, FT \\
\hline At3g5II40 & Expressed protein \\
\hline At3g60590 & Expressed protein \\
\hline At4g33520 & Metal-transporting P-type ATPase (PAAI) \\
\hline At5g 12470 & Expressed protein \\
\hline At5g64940 & $\mathrm{ABCI}$-family protein \\
\hline \multicolumn{2}{|l|}{ Chlamydia-like } \\
\hline At $\lg 15500$ & Adenine nucleotide translocase 2 (AtNTT2) \\
\hline At lg80300 & Adenine nucleotide translocase I (AtNTTI) \\
\hline At3g26570 & Low affinity phosphate transporter (PHT2;I) \\
\hline At4g37270 & Heavy metal ATPase HMAI \\
\hline At5gl2860 & Dicarboxylate translocator I (DiTI) \\
\hline At5g64280 & Dicarboxylate translocator 2.2 (DiT2.2) \\
\hline At5g64290 & Dicarboxylate translocator 2.1 (DiT2.I) \\
\hline \multicolumn{2}{|l|}{ Other } \\
\hline At lg0I790 & Potassium transporter \\
\hline At $\lg 32080$ & Membrane protein \\
\hline At lg44920 & Expressed protein \\
\hline At $\lg 54350$ & $A B C$ transporter \\
\hline At $\lg 78560$ & Bile acid:sodium symporter \\
\hline At2g02590 & Expressed protein | (Putative small multi-drug export) \\
\hline At2g21340 & Enhanced disease susceptibility protein \\
\hline At2g26900 & Bile acid:sodium symporter \\
\hline At3g25410 & Bile acid:sodium symporter \\
\hline At $4 g 30580$ & I-Acylglycerol-3-phosphate O-acyltransferase (ATS2) \\
\hline At5g03555 & Cytosine/purines, uracil, thiamine, allantoin family permease \\
\hline At5gl3720 & Expressed protein \\
\hline At5g52540 & Expressed protein \\
\hline At5g62720 & Integral membrane HPP family protein \\
\hline \multicolumn{2}{|l|}{ Plantae-specific } \\
\hline At2g38550 & Expressed protein \\
\hline At3g57280 & Expressed protein \\
\hline At5gl7520 & Maltose transporter (MEXI) \\
\hline At5g24690 & Expressed protein \\
\hline
\end{tabular}

List of Arabidopsis thaliana chloroplast solute transporters analyzed in this study and their putative evolutionary origins.

The cyanobacterial contribution

The cyanobacterial endosymbiont putatively contributed ten solute transporters to the plastid transport system (Table 1, Figure S2 in Additional data file 1). These proteins include trigalactosyldiacylglycerol 1 (TGD1; Figure 3a), which is required for integrating the prokaryotic (that is, cyanobacterial) with the eukaryotic (that is, endoplasmic reticulum) pathway for lipid biosynthesis [24-26], the metal-transporting P-type ATPase PAA1 [27,28], and a transporter required for folate/biopterin biosynthesis [29]. The remaining seven proteins of unknown function that are localized to the chloro- plast inner membrane were included in the cyanobacterial group. Whereas the predicted secondary structure of most of these proteins indicates they represent transporters (that is, they contain at least four transmembrane domains that are connected by short loops), some, such as the ABC1-family protein At5g64940 (Figure 3b) contain only one or two predicted transmembrane domains and may thus have functions other than metabolite transport. It is also intriguing that with the exception of the PAA1 copper transporter the only cyanobacterial transport proteins apparently retained by Arabidopsis are those for which the host lacked a suitable 


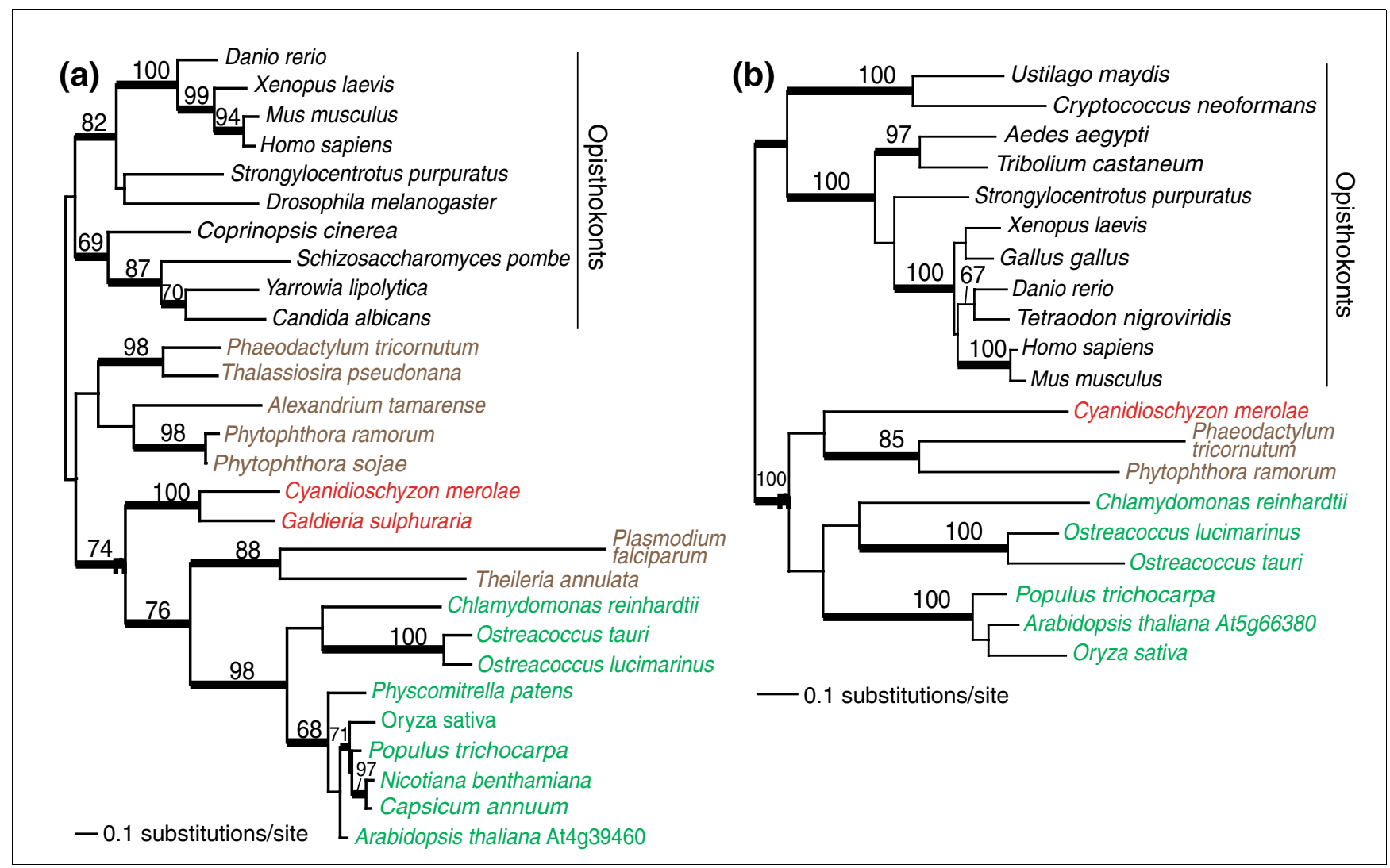

Figure 2

Plastid targeted solute transporters of putative 'Host' origin in Plantae. These are RAxML trees with the numbers above the branches inferred from a RAxML bootstrap analysis and the thick branches showing significant $(P>0.95)$ support from a Bayesian phylogenetic inference. Only bootstrap values $\geq$ $60 \%$ are shown. Branch lengths are proportional to the number of substitutions per site (see scale bars). The filled magenta circle shows the node that unites the Plantae taxa within the eukaryotic domain. The different algal groups are shown in different text colors: red for red algae, green for green algae and land plants, and brown for chromalveolates. The inclusion of chromalveolates within the Plantae is believed to reflect horizontal or endosymbiotic gene transfer events (for example, [50]). The two transporters are: (a) SAMT, S-adenosylmethionine carrier I protein; and (b) AtFOLTI, Arabidopsis thaliana folate transporter I. The name of the $A$. thaliana solute transporter used for the query is indicated for both trees shown in this figure.

replacement. For example, the initial steps of folic acid biosynthesis in plants are confined to the chloroplast; the final steps are localized in the cytosol and in mitochondria [30-32]. Plastids thus depend on an external folate supply and require an uptake system for this important metabolite. Interestingly, redundant systems for folate uptake exist in Arabidopsis chloroplasts, consisting of the cyanobacterial-derived folate transporter FT [29] and the host-derived transporter AtFOLT1 [33].

\section{'Chlamydia-like' transporters}

In addition to the host and cyanobacteria, a third significant contributor to the Plantae plastid solute transport system is the Chlamydiae. A surprisingly high number (four) of plastid envelope membrane transporters have been contributed by these prokaryotes. The presence of plant-like genes in Chlamydia has been noted in the past, sparking debate over whether their presence indicated a transfer from the ancestral plant to Chlamydia, an evolutionary relationship between cyanobacteria and Chlamydia, or a horizontal gene transfer
(HGT) from a chlamydial parasite to the plant ancestor [3436]. Phylogenetic analysis of plastid, Chlamydiae, and Rickettsiae ADP/ATP translocases [36] supports an ancient Chlamydia-to-Plantae direction of transfer. This explanation for the origin of the ADP/ATP translocase gene (and other Chlamydial-like genes) in Plantae was strongly supported by the phylogenomic analysis of Huang and Gogarten [21]. We found a monophyletic relationship between the AtNTT1 and AtNTT2 (the Arabidopsis plastid ADP/ATP translocases) and Chlamydiae ADP/ATP translocases (Figure 4a) [37,38]. In addition, the copper transporter heavy metal ATPase 1 (HMA1; Figure 4b), the dicarboxylate translocators (DiTs) DiT1, DiT2.1, and DiT2.2, and the low affinity phosphate transporter PHT2;1 (see Figure $\mathrm{S}_{3}$ in Additional data file 1 and [21]) apparently has a chlamydial origin in Plantae. All of these trees provide bootstrap (except for the DiT tree) support for the monophyly of the 'Chlamydia-like' and plastid transporters. In the case of HMA1 there are two ancient paralogs in plants, one of cyanobacterial likely endosymbiotic origin and one from a Chlamydia-like source that is shared 


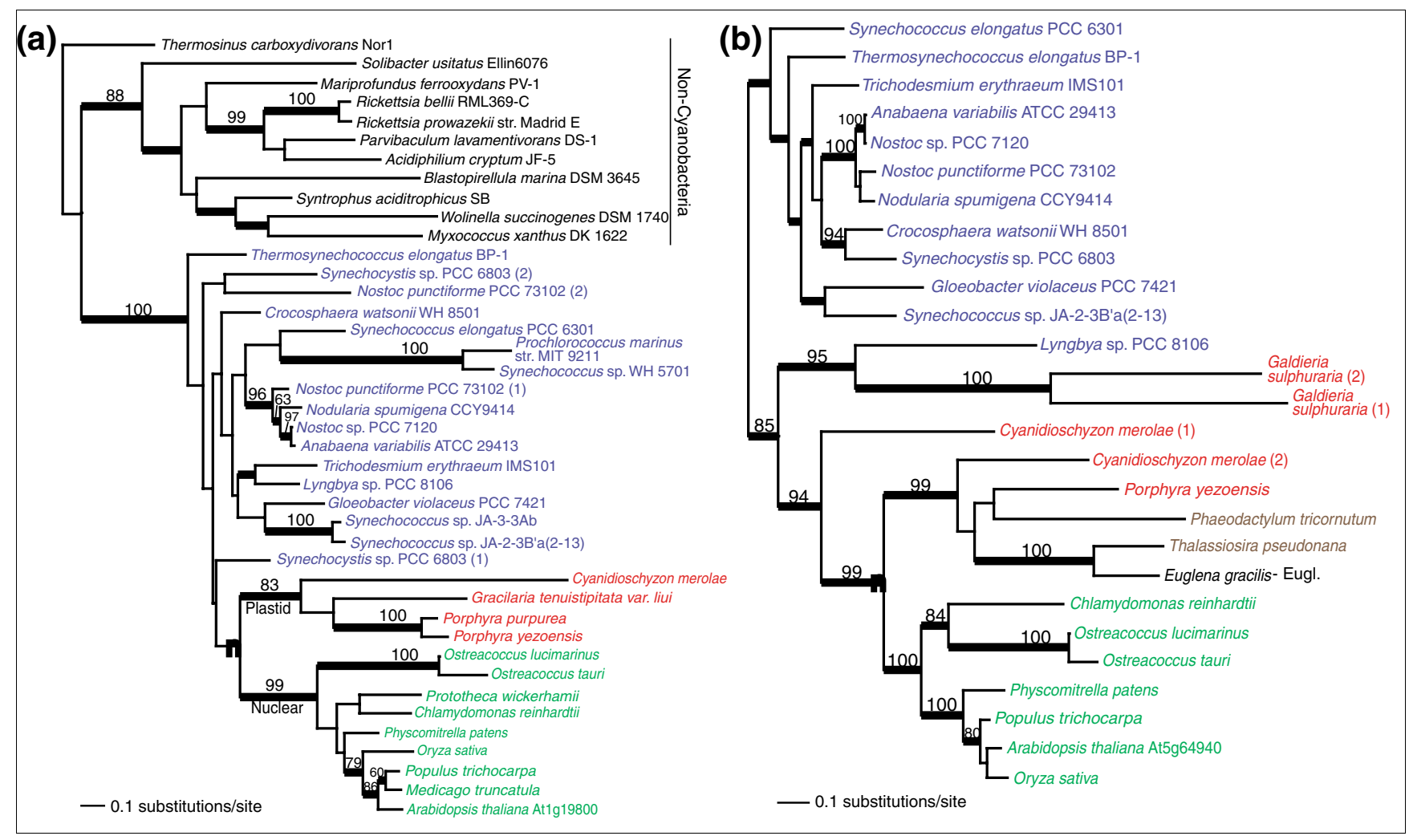

Figure 3

Plastid targeted solute transporters of putative 'Cyanobacterial' (that is, plastid endosymbiont) origin in Plantae. For details of tree building see Figure 2. The filled magenta circle shows the node that unites the Plantae taxa as sister to cyanobacteria. The different photosynthetic groups are shown in different text colors: blue for cyanobacteria, red for red algae, green for green algae and land plants, and brown for chromalveolates. The inclusion of chromalveolates or Euglenozoa (Eugl.) within the Plantae is believed to reflect horizontal or endosymbiotic gene transfer events (for example, [50]). The two transporters are: (a) TGDI, trigalactosyldiacylglycerol I, lipid transporter; and (b) ABCl-family transporter protein. The name of the A. thaliana solute transporter used for the query is indicated for both trees shown in this figure.

with red and green algae. The DiTs [39] are present only in green algae, plants, and bacteria (that is, not in red algae). Whereas genomic data for glaucophytes are not yet available, transport experiments using isolated Cyanophora cyanelles showed that this glaucophyte uses a transport system for glutamine and 2-oxoglutarate that is distinct from green plant DiTs [40]. Taken together, these data indicate that 'Chlamydia-like' dicarboxylate translocators have likely been lost from red algae and glaucophytes. An alternative explanation is that the gene was acquired by the green lineage after the split of Chlorophyta and Rhodophyta. A DiT2 gene was also found in the dinoflagellates Amphidinium carterae and Heterocapsa triquetra, which likely originated from an independent HGT. Several 'green' genes have been found in dinoflagellates and other chromalveolates that could have either originated from multiple independent HGTs or an ancient green algal endosymbiosis (for discussion, see [41]).

In summary, it is surprising that bacteria not putatively involved in the endosymbiosis contributed $8 \%$ of the transporters that we have identified. When one considers the functions of these transporters, the chlamydial contribution becomes more important. HMA1 increases copper and/or zinc transport into the plastid under conditions of high light, facilitating the production of copper/zinc superoxide dismutase (CuZnSOD), which protects the plant from superoxide radicals produced under high light conditions $[42,43]$. PHT2;1, a phosphate transporter, controls phosphate allocation under conditions of phosphate-starvation [44]. The DiT transporters are involved in assimilating nitrogen and recovering carbon lost to photorespiration, a process that is initiated by the oxygenation reaction of Rubisco that primarily occurs under conditions when a high $\mathrm{O}_{2}: \mathrm{CO}_{2}$ ratio is present in the vicinity of Rubisco. Mutants lacking these transporters are unable to survive in ambient $\mathrm{CO}_{2}$ concentrations $[17,45,46]$. Finally, the AtNTT1 and AtNTT2 transporters are required for ATP import into the plastid during the dark (that is, in the absence of photosynthetic ATP production), particularly during lipid and chlorophyll biosynthesis. Although AtNTT2 mutants are still capable of producing lipids, indicating that the plastid has an alternative method for generating the ATP required for lipid biosynthesis, the production is significantly reduced and mutant plants have a sharply reduced growth rate [16]. Arabidopsis mutants deficient in both 


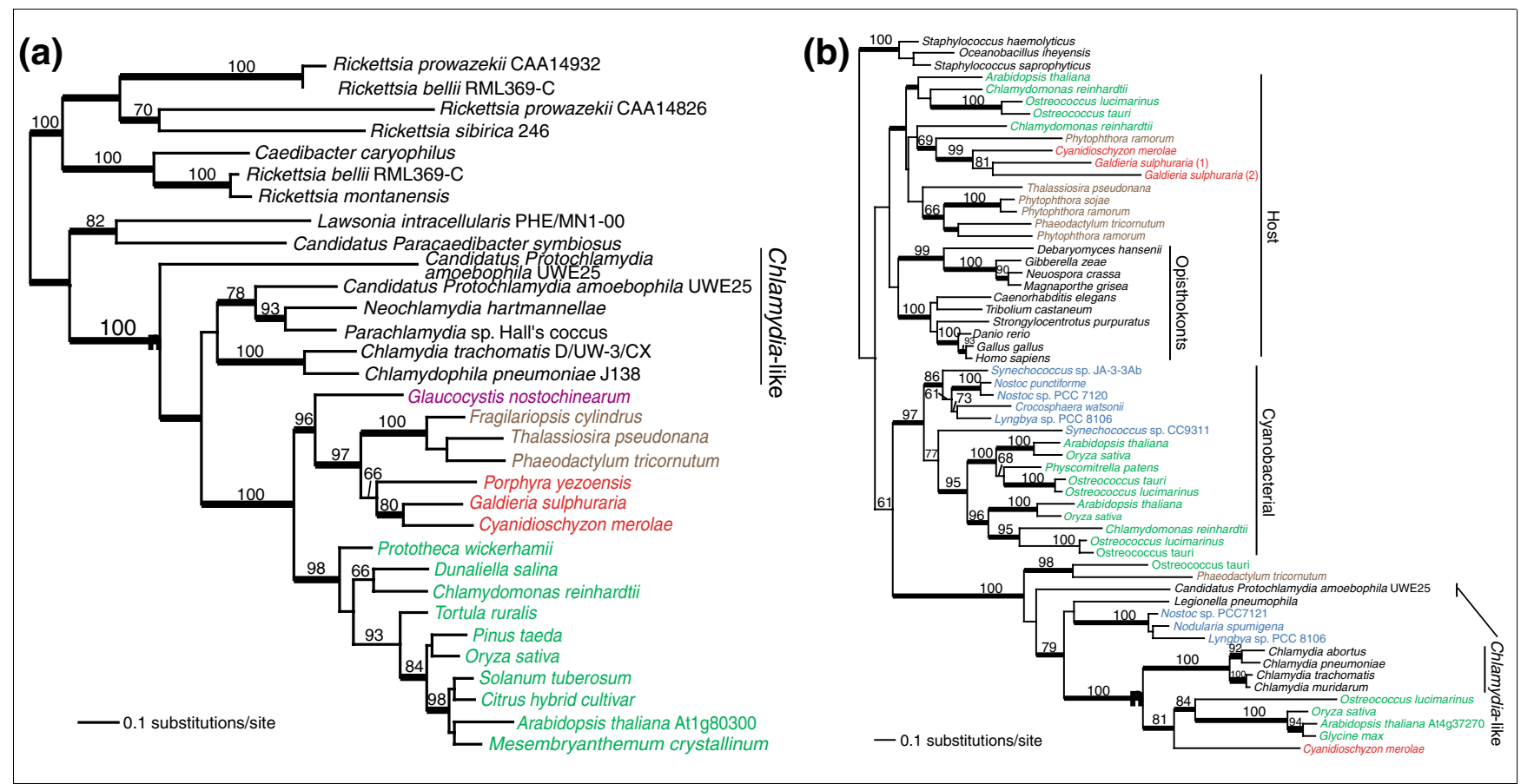

Figure 4

Plastid targeted solute transporters of putative 'Chlamydia-like' origin in Plantae. For details of tree building see Figure 2. The filled magenta circle shows the node that unites chlamydial taxa with plastid targeted Plantae transporters. The different photosynthetic groups are shown in different text colors: blue for cyanobacteria, red for red algae, green for green algae and land plants, magenta for glaucophytes, and brown for chromalveolates. The inclusion of chromalveolates within the Plantae is believed to reflect horizontal or endosymbiotic gene transfer events (for example, [50]). The two transporters are: (a) ADP/ATP translocater; and (b) heavy metal ATPase (HMAI) copper transporter. The name of the $A$. thaliana solute transporter used for the query is indicated for both trees shown in this figure.

AtNTT1 and AtNTT2 develop necrotic lesions when grown under short days, accumulate $\mathrm{H}_{2} \mathrm{O}_{2}$, and, strikingly, show constitutive expression of CuZnSOD2 and ascorbate peroxidase [47]. The phenotype of the mutant was linked to reduced magnesium chelatase activity and it was concluded that ATP import into plastids in the dark is required for chlorophyll biosynthesis and for preventing photooxidative damage [47]. The import of ATP into plastids in the dark is thus clearly a case in which the endosymbiont benefits from host metabolism. The ancient origin of these transporters in the tree of photosynthetic eukaryotes (Figure 4a) is indicative of an essential role of this uptake system in the formation of the endosymbiosis. With the exception of the DiT translocators, each of these transporters appear to perform somewhat redundant functions (that is, copper and phosphate transport) but in a way that permits the plant to adapt to stresses involved in life on the land (that is, high light and $\mathrm{O}_{2}$ levels or low phosphate availability). This may explain why the genes encoding these four plastid transporters have been retained in the Arabidopsis genome.

How the 'Chlamydia-like' genes entered into the Plantae ancestor is unclear but it is possible that both the cyanobacterial endosymbiont and chlamydial parasites may have co-existed in the cell. Many environmental Chlamydia are known today that are broadly distributed in animals and protists [48]. The co-existence of these two distinct prokaryotes may have provided the genetic 'toolkit' to make permanent the endosymbiosis with gene transfer from each cell providing essential functions for endosymbiont utilization. An alternative explanation is that the cyanobacterial endosymbiont was itself highly chimeric (that is, the 'fluid chromosome model') [49] and contained genes of chlamydial origin that had been gathered through HGT. Although possible, this scenario seems less plausible because it invokes, for example, the presence of an ADP/ATP translocator (a gene typical for 'energy parasites' such as Rickettsiae) in the genome of an oxygenic photosynthetic cell that is unlikely to encounter high concentrations of ATP in the surrounding environment; that is, it is absent from all studied cyanobacteria. Additional discussion of these issues can be found in Huang and Gogarten [21].

\section{'Other' and 'Plantae-specific' transporters}

We were unable to conclusively determine the origin of 18 transport proteins. Fourteen of these data sets resulted in PHYML trees in which the Plantae transporters were rooted within prokaryotes but without bootstrap support for a specific affiliation. An excellent example is provided by At1g32080 (Figure 5a), which is a putative membrane protein conserved among Plantae, chromalveolates, and a diverse set of Eubacteria and Archaea (that is, the Thermococcus and 
Pyrococcus clade). Although the prokaryotic source of this gene in Plantae is unclear with the available data, the eukaryotic clade is clearly monophyletic, which is consistent with a single gene origin in the Plantae ancestor and, thereafter, transfer to chromalveolates (for example, diatoms in this tree) via secondary endosymbiotic gene transfer [50]. The unresolved provenance of At1g32080 and the 'Other' set of transporters in Plantae can be explained by pervasive HGT followed by full or partial gene replacement or differential gene loss among prokaryotes that has erased the ancient phylogenetic signal. Alternatively, these results may indicate erratic rates of sequence divergence that make it impossible to model protein evolution for these sequences. Given the growing evidence, however, for recurring HGT among bacteria [51], it is likely that genes in the 'Other' category have reticulate evolutionary histories. In this regard it is noteworthy that the likely frequent HGTs seen in Figure 5a among prokaryotes and other genes in the 'Other' category contrasts starkly with the apparent single origin and vertical inheritance in Plantae (for example, At4g30580, At5g13720, At5g52540, At5g62720; Figure S4 in Additional data file 1). This result suggests a clear difference in rates of HGT for these genes with elevated rates in prokaryotes relative to eukaryotes.

Of the remaining transporters, four fell in the 'Plantae-specific' category because they lacked identifiable homologs outside of this supergroup and may simply be too divergent to determine their origin. This includes At5g24690 (Figure 5b, a hypothetical expressed protein) and the plastidic maltose exporter MEX1. The latter is required for export of maltose resulting from starch breakdown from plastids at night in green plants (Figure $\mathrm{S}_{5}$ in Additional data file 1). Storage of starch inside the chloroplast is exclusively found in the green linage. Therefore, MEX1 has likely co-evolved with plastidbased starch biosynthesis and breakdown since it can be detected only in members of the Viridiplantae with one gene found in the dinoflagellate Karlodinium micrum, which, as described above for Dit2, likely has resulted from a HGT.

\section{Conclusion}

Here we determined the phylogeny of 83 Arabidopsis plastid solute transporters to determine whether they are of endosymbiotic origin from the captured cyanobacterium, of host origin, or of a 'mixed' origin from both of these sources. Our analysis has afforded a rare look at early, critical events in primary plastid evolution and support the notion that integration of plastid-host metabolism was primarily driven by hostderived transporters with important contributions coming from the cyanobacterial endosymbiont and Chlamydia-like bacteria. Another class of proteins of currently unknown origin included plant specific transporters such as MEX1. Despite the power of our comparative approach, our work has some important limitations. One is that because we used the Arabidopsis transporter set, we most certainly have missed a number of Plantae transporters that are specific to red or green algae and have been lost from the Arabidopsis genome. In addition, we lack significant data from glaucophytes, but the upcoming Cyanophora paradoxa (glaucophyte) nuclear genome sequence [52] will allow us to incorporate this lineage into future inferences about transporter evolution. It is reasonable to assume, however, given the wealth of data supporting Plantae monophyly [2-4,7], that our inferences regarding the red and green lineages also apply to their glaucophyte sisters. Despite these limitations and the fact that phylogenetic signal is imperfectly maintained over a billion years of evolution, our comprehensive analysis of the chloroplast solute transport system will likely hold up and can be further tested as other genome sequences become available.

\section{Materials and methods Initial transporter analyses}

As a starting point for the compilation of a conservative set of predicted or confirmed plastid envelope membrane transporters, we used a previously published list of 137 plastid-targeted membrane proteins that was based on predicted plastid localization and classification by the transporter classification system [10]. This list was manually curated to remove proteins from the list if published evidence indicated that they were localized to a cellular location other than chloroplasts, if they represented membrane-bound enzymes, or if they were annotated as components of the TIC/TOC protein import apparatus, the photosynthetic machinery of the thylakoid membrane, or the Sec or Tat protein targeting pathways. This curated list of candidate genes was updated and amended with recently published chloroplast envelope membrane transporters, such as AtFOLT1, a plastid localized transporter belonging to the mitochondrial carrier family that does not contain a plastid targeting signal [33] and was thus not included in previous lists. The final list contained $83 \mathrm{~A}$. thaliana predicted or confirmed chloroplast solute transporters.

The sequence for each protein was obtained from The Arabidopsis Information Resource website [53]. These protein sequences were used as queries in blastp and tblastn searches of the NCBI Database [54], the plant and algal genomes available through the Joint Genome Institute [55], the Cyanidioschyzon merolae Genome Project website [56], the Galdieria sulphuraria Genome Project website [57], and Dragonblast V2.1 (SE Ruemmele, unpublished data), a web based database in the DB lab that contains EST datasets for several chromalveolates, Plantae, excavates, Rhizaria, and Amoebozoa. We used the predicted protein sequences for the following species for our analysis whenever available: Arabidopsis thaliana, Oryza sativa, Physcomitrella patens, Chlamydomonas reinhardtii, Ostreococcus tauri, Ostreococcus lucimarinus, Cyanidioschyzon merolae, Galdieria sulphuraria, Cyanophora paradoxa, Dictyostelium discoideum, Strongylocentrotus purpuratus, Xenopus lae- 


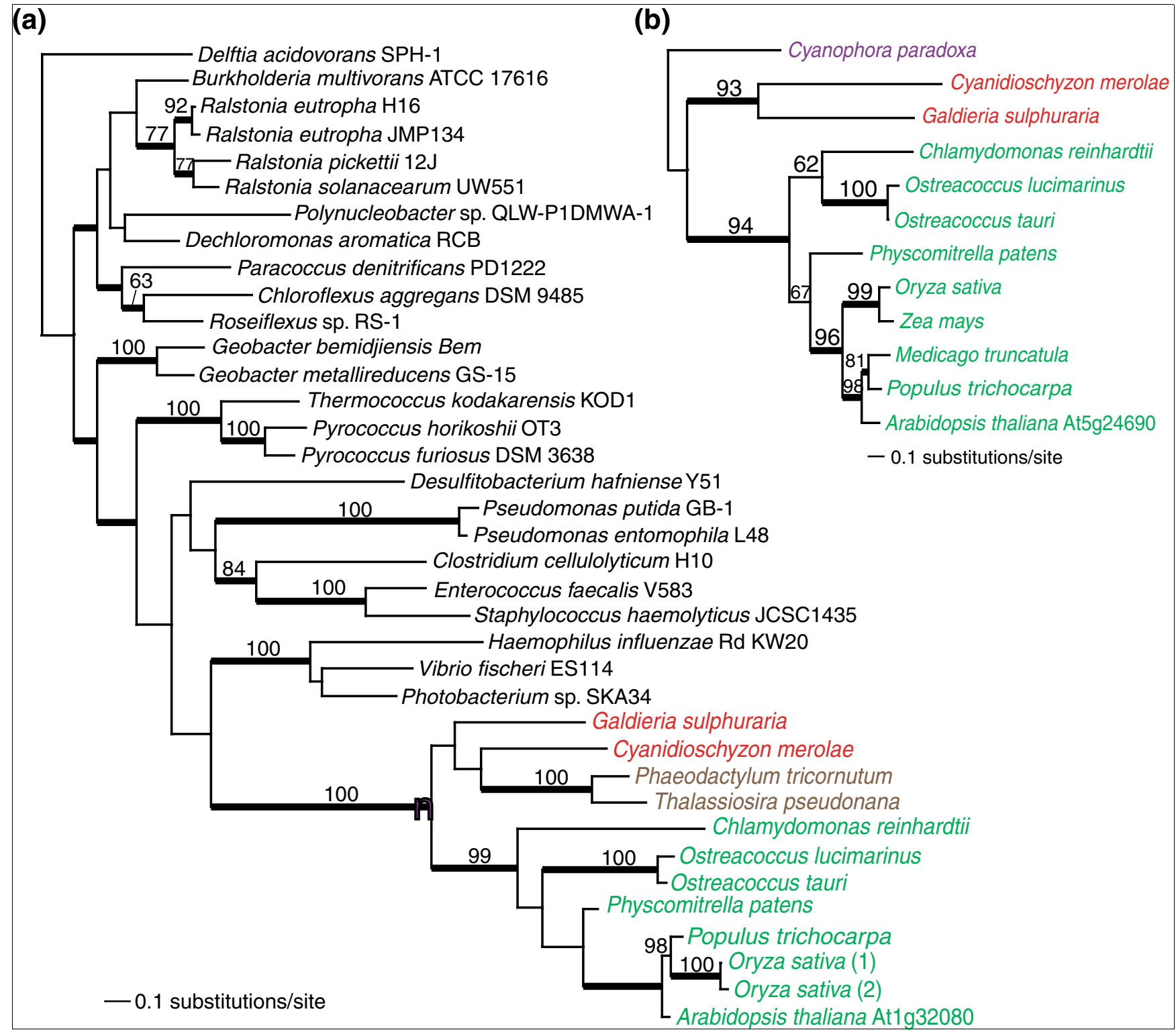

\section{Figure 5}

Plastid targeted solute transporters of 'Other' or 'Plantae-specific' origin in Plantae. For details of tree building see Figure 2. The filled magenta circle shows the node that unites the Plantae taxa. The different algal groups are shown in different text colors: red for red algae, green for green algae and land plants, magenta for glaucophytes, and brown for chromalveolates. The inclusion of chromalveolates within the Plantae is believed to reflect horizontal or endosymbiotic gene transfer events (for example, [50]). The different transporters are: (a) transporter in the 'Other' category: putative membrane protein; and (b) transporter in the 'Plantae-specific' category: hypothetical expressed protein. The name of the A. thaliana solute transporter used for the query is indicated for both trees shown in this figure.

vis, Danio rerio, Mus musculus, Canis familiaris, and Homo sapiens. In addition, we included at least one insect, three fungal species, and a broad range of Bacteria and Archaea in our analysis. The BLAST searches used an e-value cut-off < 10-5. If a translated EST sequence was not available, the nucleotide sequence was translated over six frames using the ExPASy translate tool [58]. The resulting protein sequences were used in a BLAST search against the NCBI protein database to ensure the correct translation was obtained.
We used the ClustalW feature included with BioEdit V7.0.5.3 to generate protein alignments [59]. Alignments were visually inspected and manually corrected if necessary. Trees were generated under maximum likelihood using PHYML V2.4.4 utilizing the WAG model of amino acid substitution and estimating both the proportion of invariable sites and the alpha parameter (that is, WAG $+\mathrm{I}+\Gamma)[60]$. We performed nonparametric bootstrap analysis with 100 replicates for each PHYML analysis. The resulting trees were analyzed to deter- 
mine the origin of the transporter in Arabidopsis and other Plantae.

The designation of gene origin was done as follows. When the Plantae solute transporter formed a well-supported (usually $>70 \%$ bootstrap support) monophyletic group with homologs in opisthokonts (that is, animals and fungi) and secondarily with other eukaryotes such as excavates and chromalveolates (if present), then it was classified as having a 'Host' origin. Under this scheme, no bacterial sequences interrupted the eukaryotic domain. 'Cyanobacterial' or 'Chlamydia-like' origin was inferred if the Plantae sequence formed a monophyletic group with protein sequences from either of these lineages with strong bootstrap support. Other bacterial or eukaryotic sequences could (not necessarily) be in these trees but there had to be a robust separation of the Plantae + cyanobacteria or Chlamydia-like clade from all other homologs. We had two other categories of gene origin that likely reflected a lack of phylogenetic resolution or pervasive HGT among taxa that defied a clear inference of origin. The first was the 'Other' category in which the Plantae transporter formed a well-supported monophyletic clade but its position relative to available bacterial data was unresolved, thereby not allowing us to identify the donor taxon. The second, 'Plantae-specific', was for transporters that had no significant hits to sequences in GenBank or other databases and appeared to be limited to the Plantae. All of the transporter PHYML bootstrap trees are available in Additional data file 1. The protein alignments are available in the download section of the Bhattacharya Lab website [61].

\section{Detailed phylogenetic analyses}

For eight representative transporters from the five categories described above we inferred a maximum likelihood phylogeny using RAxML (RAxML-VI-HPC, v2.2.1) [62] and the WAG $+\Gamma$ evolutionary model. The specific transporters were: 'Host' - At4g3946o (SAMT, S-adenosylmethionine carrier 1 protein, 236 amino acids), At5g66380 (AtFOLT1, Arabidopsis thaliana folate transporter 1, 268 amino acids); 'Cyanobacterial' - At1g1980o (TGD1, trigalactosyldiacylglycerol 1, lipid transporter, 234 amino acids), At5g64940 (ABC transporter protein, 487 amino acids); 'Chlamydia-like' At1g15500 (chloroplast ADP, ATP carrier protein 2, 436 amino acids), At4g37270 (HMA1, copper exporting ATPase, 444 amino acids); 'Other' - At1g32080 (putative membrane protein, 211 amino acids); 'Plantae-specific' - At5g2469o (hypothetical expressed protein, 274 amino acids). These detailed analyses used a random starting tree (one round of taxon addition) and the rapid hill-climbing algorithm (that is, option - $\mathrm{f} d$ in RAxML). To generate bootstrap values for these phylogenies, we used RAxML with the same settings and 100 replications. In addition, we used Bayesian inference (MrBayes V3.ob4) [63] with each of the eight data sets using the WAG $+\mathrm{I}+\Gamma$ model to calculate posterior probabilities for nodes in the RAxML trees. Metropolis-coupled Markov chain Monte Carlo from a random starting tree was used in this analysis with two independent runs (that is, nrun $=2$ command) and 1 cold and 3 heated chains. The Bayesian analyses were run for two million generations each with trees sampled every 10oth generation. To increase the probability of chain convergence, we sampled trees after the standard deviation values of the two runs were $<0.01$ to calculate the posterior probabilities. We also ran the Bayesian analysis for the remaining two putative 'Chlamydia-like' genes in Plantae (dicarboxylate translocators DiT1, DiT2.1, and DiT2.2, and the phosphate transporter PHT2;1) to assess the topologies. We incorporated a representative diversity of available sequences in all of these trees.

\section{Abbreviations}

AtFOLT, A. thaliana folate transporter; AtNTT, A. thaliana ADP/ATP translocase; DiT, dicarboxylate translocator; EST, expressed sequence tag; HGT, horizontal gene transfer; HMA, heavy metal ATPase; TGD, trigalactosyldiacylglycerol.

\section{Authors' contributions}

APMW and ML gathered and prepared the Arabidopsis plastid transporter data for downstream bioinformatic analyses. HMT did the subsequent database searches and built the initial phylogenetic trees. DB was responsible for the final phylogenetic trees presented in the manuscript figures. HMT wrote the initial draft of the manuscript. APMW and DB conceived of and supervised this study and prepared the final manuscript. All authors read and approved the final manuscript.

\section{Additional data files}

The following additional data are available with the online version of this paper. Additional data file 1 shows trees of all remaining plastid transporters analyzed in this study

\section{Acknowledgements}

This work was supported by NSF Award EF-0332882 to APMW and NIH Award ROI ESOI3679-0I and NSF Award EF-043III7 to DB.

\section{References}

I. Yoon HS, Hackett JD, Ciniglia C, Pinto G, Bhattacharya D: A molecular timeline for the origin of photosynthetic eukaryotes. Mol Biol Evol 2004, 2 I :809-8I8.

2. Rodriguez-Ezpeleta N, Brinkmann H, Burey SC, Roure B, Burger G, Loffelhardt W, Bohnert HJ, Philippe H, Lang BF: Monophyly of primary photosynthetic eukaryotes: green plants, red algae, and glaucophytes. Curr Biol 2005, I5:1325-1330.

3. Weber AP, Linka M, Bhattacharya $D$ : Single, ancient origin of a plastid metabolite translocator family in Plantae from an endomembrane-derived ancestor. Eukaryot Cell 2006 5:609-6I2.

4. Reyes-Prieto A, Bhattacharya D: Phylogeny of Calvin cycle enzymes supports Plantae monophyly. Mol Phylogenet Evol .

5. Bhattacharya D, Yoon HS, Hackett JD: Photosynthetic eukaryotes unite: endosymbiosis connects the dots. Bioessays 2004, 26:50-60. 
6. Cavalier-Smith T, Allsopp MT, Chao EE: Chimeric conundra: are nucleomorphs and chromists monophyletic or polyphyletic? Proc Natl Acad Sci USA 1994, 9 I: I I 368-I I 372.

7. McFadden GI: Endosymbiosis and evolution of the plant cell. Curr Opin Plant Biol 1999, 2:513-519.

8. Marin B, Nowack EC, Melkonian M: A plastid in the making: evidence for a second primary endosymbiosis. Protist 2005 , I 56:425-432.

9. Yoon HS, Reyes-Prieto A, Melkonian M, Bhattacharya D: Minimal plastid genome evolution in the Paulinella endosymbiont. Curr Biol 2006, 16:R670-672.

10. Weber AP, Schwacke R, Flügge UI: Solute transporters of the plastid envelope membrane. Annu Rev Plant Biol 2005, 56:133-164.

II. Gutensohn M, Fan E, Frielingsdorf S, Hanner P, Hou B, Hust B, Klösgen RB: Toc, Tic, Tat et al.: structure and function of protein transport machineries in chloroplasts. J Plant Physiol 2006, 1 63:333-347.

12. Timmis JN, Ayliffe MA, Huang CY, Martin W: Endosymbiotic gene transfer: organelle genomes forge eukaryotic chromosomes. Nat Rev Genet 2004, 5: I 23-135.

13. Okamoto $N$, Inouye I: A secondary symbiosis in progress? Science 2005, 310:287.

14. Johnson PW, Hargraves PE, Sieburth JM: Ultrastructure and ecology of Calycomonas ovalis Wulff, (Chrysophyceae) and its redescription as a testate rhizopod, Paulinella ovalis n. comb. (Filosea: Euglyphina). J Protozool 1919, 35:618-626.

I5. Raven JA, Evans MCW, Korb RE: The role of trace metals in photosynthetic electron transport in $\mathrm{O}_{2}$-evolving organisms. Photosynthesis Res 1999, 60:1 I I-I50.

16. Reiser J, Linka N, Lemke L, Jeblick W, Neuhaus HE: Molecular physiological analysis of the two plastidic ATPIADP transporters from Arabidopsis. Plant Physiol 2004, I 36:3524-3536.

17. Renné $P$, Dressen U, Hebbeker U, Hille $D$, Flügge UI, Westhoff $P$ Weber AP: The Arabidopsis mutant dct is deficient in the plastidic glutamate/malate translocator DiT2. Plant J 2003, 35:316-331.

18. Ferro M, Salvi D, Brugiere S, Miras S, Kowalski S, Louwagie M, Garin J, Joyard J, Rolland N: Proteomics of the chloroplast envelope membranes from Arabidopsis thaliana. Mol Cell Proteomics 2003 2:325-345.

19. Weber AP: Solute transporters as connecting elements between cytosol and plastid stroma. Curr Opin Plant Biol 2004, 7:247-253

20. Weber APM, Fischer K: Making the connections - the crucial role of metabolite transporters at the interface between chloroplast and cytosol. FEBS Lett 2007, 58 I:2215-2222.

21. Huang J, Gogarten JP: Did an ancient chlamydial endosymbiosis facilitate the establishment of primary plastids? Genome Biol 2007, 8:R99.

22. Archibald JM, Rogers MB, Toop M, Ishida K, Keeling PJ: Lateral gene transfer and the evolution of plastid-targeted proteins in the secondary plastid-containing alga Bigelowiella natans. Proc Natl Acad Sci USA 2003, I 00:7678-7683.

23. Hackett JD, Yoon HS, Li S, Reyes-Prieto A, Rümmele SE, Bhattacharya D: Phylogenomic analysis supports the monophyly of cryptophytes and haptophytes and the association of 'Rhizaria' with Chromalveolates. Mol Biol Evol 2007, 24: I702-1713.

24. Benning C, Xu C, Awai K: Non-vesicular and vesicular lipid trafficking involving plastids. Curr Opin Plant Biol 2006, 9:24I-247.

25. Awai K, Xu C, Lu B, Benning C: Lipid trafficking between the endoplasmic reticulum and the chloroplast. Biochem Soc Trans 2006, 34:395-398.

26. Xu C, Fan J, Riekhof W, Froehlich JE, Benning C: A permease-like protein involved in ER to thylakoid lipid transfer in Arabidopsis. EMBO J 2003, 22:2370-2379.

27. Shikanai T, Müller-Moulé P, Munekage Y, Niyogi KK, Pilon M: PAA I, a P-type ATPase of Arabidopsis, functions in copper transport in chloroplasts. Plant Cell 2003, I 5:1333-1346.

28. Abdel-Ghany SE, Müller-Moulé P, Niyogi KK, Pilon M, Shikanai T: Two P-type ATPases are required for copper delivery in Arabidopsis thaliana chloroplasts. Plant Cell 2005, I 7: |233- | 25 |

29. Klaus SM, Kunji ER, Bozzo GG, Noiriel A, de la Garza RD, Basset GJ, Ravanel S, Rébeillé F, Gregory JF 3rd, Hanson AD: Higher plant plastids and cyanobacteria have folate carriers related to those of trypanosomatids. J Biol Chem 2005, 280:38457-38463.

30. Sahr T, Ravanel S, Basset G, Nichols BP, Hanson AD, Rébeillé F: Folate synthesis in plants: purification, kinetic properties, and inhibition of aminodeoxychorismate synthase. Biochem J 2006, 396:157-162

31. Basset GJ, Ravanel S, Quinlivan EP, White R, Giovannoni J], Rébeillé F, Nichols BP, Shinozaki K, Seki M, Gregory JF 3rd, et al.: Folate synthesis in plants: the last step of the p-aminobenzoate branch is catalyzed by a plastidial aminodeoxychorismate lyase. Plant J 2004, 40:453-46I.

32. Basset GJ, Quinlivan EP, Ravanel S, Rébeillé F, Nichols BP, Shinozaki K, Seki M, Adams-Phillips LC, Giovannoni J], Gregory JF 3rd, et al.: Folate synthesis in plants: the p-aminobenzoate branch is initiated by a bifunctional PabA-PabB protein that is targeted to plastids. Proc Natl Acad Sci USA 2004, I 0 I: | 496- I 50 I.

33. Bedhomme M, Hoffmann M, McCarthy EA, Gambonnet B, Moran RG, Rébeillé F, Ravanel S: Folate metabolism in plants: an Arabidopsis homolog of the mammalian mitochondrial folate transporter mediates folate import into chloroplasts. J Biol Chem 2005, 280:34823-34831.

34. Brinkman FS, Blanchard JL, Cherkasov A, Av-Gay Y, Brunham RC, Fernandez RC, Finlay BB, Otto SP, Ouellette BF, Keeling PJ, et al.: Evidence that plant-like genes in Chlamydia species reflect an ancestral relationship between Chlamydiaceae, cyanobacteria, and the chloroplast. Genome Res 2002, I 2: I I 59-I I67.

35. Wolf YI, Aravind L, Koonin EV: Rickettsiae and Chlamydiae: evidence of horizontal gene transfer and gene exchange. Trends Genet 1999, I 5: 173-175.

36. Schmitz-Esser S, Linka N, Collingro A, Beier CL, Neuhaus HE, Wagner M, Horn M: ATPIADP translocases: a common feature of obligate intracellular amoebal symbionts related to Chlamydiae and Rickettsiae. J Bacteriol 2004, I 86:683-69|

37. Greub G, Raoult D: History of the ADP/ATP-translocaseencoding gene, a parasitism gene transferred from a Chlamydiales ancestor to plants I billion years ago. Applied Environmental Microbiol 2003, 69:5530-5535.

38. Linka N, Hurka $H$, Lang BF, Burger $G$, Winkler $H H$, Stamme $C$ Urbany C, Seil I, Kusch J, Neuhaus HE: Phylogenetic relationships of non-mitochondrial nucleotide transport proteins in bacteria and eukaryotes. Gene 2003, 306:27-35.

39. Weber A, Flügge UI: Interaction of cytosolic and plastidic nitrogen metabolism in plants. J Exp Bot 2002, 53:865-874.

40. Kloos K, Schlichting R, Zimmer W, Bothe H: Glutamine and glutamate transport in Cyanophora paradoxa. Bot Acta 1993, I 06: $435-440$.

4I. Nosenko T, Lidie KL, Van Dolah FM, Lindquist E, Cheng JF, Bhattacharya D: Chimeric plastid proteome in the Florida "red tide" dinoflagellate Karenia brevis. Mol Biol Evol 2006, 23:2026-2038.

42. Seigneurin-Berny D, Gravot A, Auroy P, Mazard C, Kraut A, Finazzi G, Grunwald D, Rappaport F, Vavasseur A, Joyard J, et al.: HMA I, a new Cu-ATPase of the chloroplast envelope, is essential for growth under adverse light conditions. J Biol Chem 2006, 281:2882-2892.

43. Lee YP, Kim SH, Bang JW, Lee HS, Kwak SS, Kwon SY: Enhanced tolerance to oxidative stress in transgenic tobacco plants expressing three antioxidant enzymes in chloroplasts. Plant Cell Rep 2007, 26:59I-598.

44. Versaw WK, Harrison MJ: A chloroplast phosphate transporter, PHT2; I, influences allocation of phosphate within the plant and phosphate-starvation responses. Plant Cell 2002, | 4: 1751 - | 766.

45. Schneidereit J, Häusler RE, Fiene G, Kaiser WM, Weber AP: Antisense repression reveals a crucial role of the plastidic 2 oxoglutarate/malate translocator DiTI at the interface between carbon and nitrogen metabolism. Plant J 2006, 45:206-224.

46. Reumann S, Weber APM: Plant peroxisomes respire in the light: Some gaps of the photorespiratory $C_{2}$ cycle have become filled - others remain. Biochim Biophys Acta 2006, I 763: I 496-I 510.

47. Reinhold T, Alawady A, Grimm B, Beran KC, Jahns P, Conrath U Bauer J, Reiser J, Melzer M, Jeblick W, et al:: Limitation of nocturnal import of ATP into Arabidopsis chloroplasts leads to photooxidative damage. Plant / 2007, 50:293-304.

48. Molmeret M, Horn M, Wagner M, Santic M, Abu Kwaik Y: Amoebae as training grounds for intracellular bacterial pathogens. Applied Environmental Microbiol 2005, 7 I:20-28.

49. Embley TM, Martin W: Eukaryotic evolution, changes and challenges. Nature 2006, 440:623-630.

50. Li S, Nosenko T, Hackett JD, Bhattacharya D: Phylogenomic analysis identifies red algal genes of endosymbiotic origin in the chromalveolates. Mol Biol Evol 2006, 23:663-674. 
51. Dagan T, Martin W: Ancestral genome sizes specify the minimum rate of lateral gene transfer during prokaryote evolution. Proc Natl Acad Sci USA 2007, 1 04:870-875.

52. Cyanophora Genome Project [http://www.biology.uiowa.edu/ cyanophora/]

53. The Arabidopsis Information Resource [http://www.arabidop sis.org/index.jsp]

54. National Center for Biotechnology Information [http:// www.ncbi.nlm.nih.gov/]

55. Joint Genome Institute Eukaryotic Genomics Website [http://genome.jgi-psf.org/euk_home.html]

56. Cyanidioschyzon merolae Genome Project [http://mero lae.biol.s.u-tokyo.ac.jp/]

57. The Galdieria sulphuraria Genome Project [http://genom
Then ics.msu.edu/galdieria/about.html]

58. ExPASy Translate Tool [http://ca.expasy.org/tools/dna.html]

59. Hall TA: BioEdit: a user-friendly biological sequence alignment editor and analysis program for Windows 95/98/NT. Nucleic Acids Symp Ser 1999, 4 1:95-98.

60. Guindon S, Gascuel O: A simple, fast, and accurate algorithm to estimate large phylogenies by maximum likelihood. Systematic Biol 2003, 52:696-704.

61. The Bhattacharya Lab Website [http://www.biology.uiowa.edu/ debweb/downloads/index.php]

62. Stamatakis A, Ludwig T, Meier H: RAxML-III: a fast program for maximum likelihood-based inference of large phylogenetic trees. Bioinformatics 2005, 21 :456-463.

63. Huelsenbeck JP, Ronquist F: MRBAYES: Bayesian inference of phylogenetic trees. Bioinformatics 200I, I 7:754-755. 